Cinémas

Revue d'études cinématographiques

Journal of Film Studies

\title{
Document : Lettres à Léon Moussinac
}

\section{Sergueï M. Eisenstein}

Volume 11, numéro 2-3, printemps 2001

Eisenstein dans le texte

URI : https://id.erudit.org/iderudit/024851ar

DOI : https://doi.org/10.7202/024851ar

Aller au sommaire du numéro

Éditeur(s)

Cinémas

ISSN

1181-6945 (imprimé)

1705-6500 (numérique)

Découvrir la revue

Citer cet article

Eisenstein, S. M. (2001). Document : Lettres à Léon Moussinac. Cinémas, 11(2-3),

161-164. https://doi.org/10.7202/024851ar d'utilisation que vous pouvez consulter en ligne.

https://apropos.erudit.org/fr/usagers/politique-dutilisation/ 


\section{DOCUMENT}

\section{Lettres à Léon Moussinac}

\section{Sergueï M. Eisenstein}

\section{I}

16 décembre 1928 [Moscou ${ }^{1}$ ]

$[\ldots]$

Le peu de temps que j'ai pu dérober au "coupage" de mon Octobre. Du point de vue de la construction l'Octobre n'est pas impeccable du tout. C'est qu'à ce film si "populaire", si "masse" dans son intention je me suis permis de faire des essais expérimentals! Si peu qu'on les vois jusqu'à présent (l'Allemagne n'en a rien compris!) ils ont suffis pour casser la composition du film comme unité. Mais d'autre côté ils m’ont suffis à en faire des déductions allant très très loin.

Je crois que je suis près à renverser tout mon système. Thématiquement ainsi que formellement. Je crois que dans le film «de l'autre côté" du film acteur, du film manière "new reel" ainsi que du film absolu, nous allons trouver la clef vers une cinématographie pure.

Et ce qui est le plus drôle - cette cinématographie sera génétiquement idéologique, car sa matière sera l'écranisation des...

(et voici une sorte de coup de théâtre - le mot essentiel de toute cette écrivasserie me manque! mon explication devient charade. Et pas cle dictionnaire sous la main. Bien prenez le mot en allemand Begriff ou bien en russe - ce qui doit vous être plus près! - poniäatïe)

Mais il n'y a pas de Begriff (griffes!) absolus. Ils sont toujours "classiques» (du mot "classes" et non "classicisme»!)

Une telle écranisation tendancieuse est la polonaise des dieux autour de Kornilov (Octobre acte trois) qui ne sont là que pour 
démontrer l'idée de la Déité de notre point de vue. Le découronnement de la Déité. Certes de tels "nota bene» en cours d'un film exotticio-pathétique ne peuvent pas ne pas le "gâter".

Mais la perspective des moyens et des thèmes qui s'ouvrent de ce côté valent bien un film un peu raté devant le gros public snobiste! La plus grosse bêtise (que l'on a fait dans presque tous les journaux allemands!) serait de comparer Octobre à Potemkine. Octobre c'est la dénégation dialectique de Pot! Et l'intérêt principiel d'Oct. est dans les bouts et morceaux qui ne ressemblent pas au Croiseur!

$$
\text { [...] }
$$

La "proclamation" de ce que je vais "filmer le Capital" de Marx n'est aucunement un truc de réclame.

Je crois qu'en cette direction se trouve la thématique du film à venir (ou bien l'écranisation de l' "Idée du Christianisme" au point de vue bourgeois)! En tout cas dans la région philosophique. Il est bien vrai que j'aborderai ce sujet pas avant un an, un an et demi car ce terrain est purement intact. Tabula rasa. Et il faudra faire beaucoup de croquis avant de s'essayer à un thème si énorme sans le compromettre.

II

Août $1931\left(\right.$ Mexico $\left.^{2}\right)$

$[\ldots]$

Le film [Que Viva Mexico!] se fait comme production indépendante - l'argent a été trouvé par Upton Sinclair parmi ses amis et porte le caractère d'un certain protest contre les emmerdeurs hollywoodiens. La Soyouzkino nous a prolongé notre séjour à l'étranger - et le film lui revient gratuitement.

Il m'est fort chagrin de vous l'écrire mais à ce que je reçois comme information (assez régulière) de Moscou n'indique rien d'intéressant dans le domaine actuel de notre cinéma. Rien de valeur ne se fait et rien d'intéressant en projet... Je m'abstiens d'explications du fait mais du point de vue marxiste cela est exactement à quoi il fallait et faut s'attendre dans le type des conditions administratives que l'on impose à la fabrication des 
films. On a perdu la mesure et l'équilibre -- on impose au film des problèmes d'une telle forme qu'il ne peut les exprimer et accomplir - sans produire un changement à fond de ses méthodes d'expression et de tout le système cinématographique. La vieille putain fardée qu'est le cinéma actuel (y compris le nôtre — il n'est qu'un peu plus fardé... de rouge!) n'est pas à la hauteur des possibilités pour exprimer ce qu'on a à dire.

Vous connaissez mes idées sur le cinéma à venir et le cinéma intellectuel et je crois qu'avant de révolutionner le cinéma encore une fois -- nous n'aurons rien d'important dans ce domaine. Il y a déjà plus de deux ans de cette stérilité. Turksib c'est très gentil - mais d'un éclectisme et dilettantisme accompli. C'est une sorte de bouquet d'une Ligne générale et d'un Vertoff mal digéré.

Je travaille beaucoup à mon livre qui probablement sera assez intéressant - c'est un résumé théorétique de dix ans (deux piatilektas) de pratique (quand même) et d'un plan de plusieurs piatilektas en avant!

[...]

Notes : François Albera

\section{NOTES}

1. Bibliothèque Nationale de France (Arsenal), Fonds Moussinac, $n^{\circ} 008$. La lettre est écrite en français et nous avons respecté l'orthographe et l'expression de l'auteur.

2. BNF (Arsenal), fonds Moussinac, $n^{\circ}$ 026. Lettre écrite en français. 


\section{THE SURER-OEJECTIVE}

eave it to that miraculow fairy, what lice within the realm of buense concions

ole on the right road and it will move ahead. It win ||
cr and dexper and will in the end lead to inepiration "

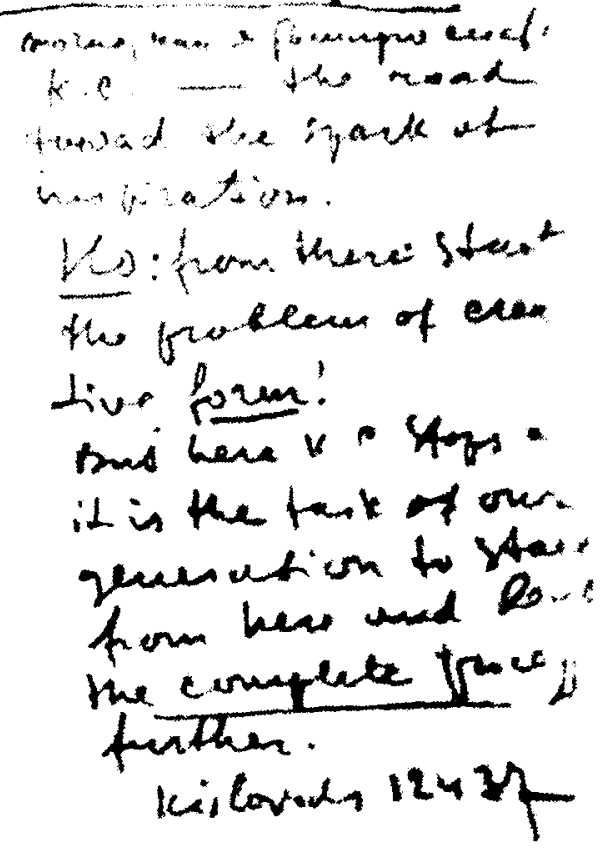

Reproduction d'une page de An Actor Prepares, de Stanislavski, avec annotation manuscrite d'Eisenstein (page 278 de l'édition française) dont les dernières lignes, commentées par Eisenstein, sont celles-ci :

"Ne comptez pas sur moi! mon "système" ne vous fabriquera jamais de l'inspiration. Tout ce qu'il peut faire, c'est lui préparer un terrain favorable.

A votre place je n'essaierais pas de poursuivre un fantôme. Laissez-en le soin à votre bonne fée, la Nature, et ne vous occupez que de ce qui se trouve dans les limites du conscient.

Lancez un rôle dans la bonne voie, et il avancera de lui-même. Il grandira et s'approfondira et finira par vous mener à l'inspiration."

Lannotation d'Eisenstein se lit comme suit :

"C'est exactement comme cela que je comprends le système de K. S[tanislavski]. - le chemin vers l'étincelle de l'inspiration.

Mais: c'est de là que part le problème de la forme créative!

Mais ici K. S. cale et c'est la tâche de notre génération de repartir de là er de propulser lensemble du processus vers l'avant.

Kislovodsk, 12.4.37" 